\title{
ALAIN DESROSIÈRES, La política de los grandes números. Editorial
} Melusina, Barcelona, 2004

Publicado en francés el año 1993, y traducido a la lengua inglesa en 1998, nos llega ahora la traducción española de este libro convertido ya en un clásico, no sólo de la historia de la estadística sino también de la sociología. Su enfoque sociológico hace de él una lectura indispensable para comprender las dimensiones sociales de las prácticas y las herramientas estadísticas. Desrosières, estadístico en el INSEE (Instituto Nacional de Estadística y Estudios Económicos) desde 1965, nos proporciona una mirada crítica, desde dentro, de esta área del conocimiento científico. Remontándose al origen de la disciplina a mediados del siglo XVII, y extendiéndose hasta los años cuarenta del siglo XX -momento en el que los instrumentos matemáticos y las estadísticas son prácticamente los que hoy conocemos- desarrolla una interesante perspectiva histórica de las teorías y las prácticas en el terreno de la estadística. Su trabajo se apoya en dos categorías de estudios históricos, los referidos a las instituciones y los sistemas estadísticos, y los referidos a la estadística matemática y las probabilidades.

La política de los grandes números condensa el extenso trabajo de Desrosières entre 1965 y 1993 , período en el que publicó numerosos artículos dedicados al estudio de la historia de la estadística, las nomenclaturas socio-profesionales, el estudio comparado de los distintos sistemas estadísticos europeos y las estadísticas sociales o industriales. Pero también refleja su participación en diversos foros de debate multidisciplinares, en los que ha colaborado con matemáticos, sociólogos, estadísticos e historiadores de la ciencia, no sólo en el INSEE o el CNRS (Centre National de Recherche Scientifique), sino sobre todo en el Seminario Histoire $d u$ calcul des probabilités et de la statistique en l'École des Hautes Études en Sciences Sociales de Paris. Se trata de un grupo de investigadores que desarrollan sus trabajos desde una perspectiva original sobre la estadística y las matemáticas sociales poco extendida fuera del ámbito francés. En España, Desrosiéres es ya bastante conocido, participó en el seminario de Estadística y Ciencias Sociales del departamento de Sociología I de la UNED, en 1997 y en las jornadas sobre Estadística y Sociedad, organizadas, en la Casa de Velázquez en el año 2000 , cuando tuvimos, además, la oportunidad de contar con otros miembros del seminario de Paris, como M. Barbut, M. Armatte, E. Brian, T. Porter, M. Mespoulet y N. Meusnier.

Es precisamente su profundo conocimiento del trabajo estadístico el que le lleva a interesarse por el origen de esas objetivaciones cuantitativas de la realidad social y la manera en que llegan a formar parte de esa realidad que al mismo tiempo pretenden describir. Si la actividad estadística produce objetos que para cumplir su cometido han de ser sólidos, estables y, en tanto que referentes de la realidad, indiscutibles, el presente libro acomete la empresa de discutir lo indiscutible, aportando un análisis profundo sobre esas construcciones sociales tan firmemente asentadas.

La sociedad utiliza cada vez más las estadísticas. El paro, los índices de precios, la tasa de natalidad y muchos otros objetos estadísticos presentes en nuestra vida cotidiana nos sirven para describir la sociedad pero también son utilizados para tomar decisiones políticas y económicas. Estos dos aspectos de la estadística, el de técnica orientada hacia el conocimiento de 
la sociedad, pero también el de instrumento indispensable para la acción política, económica y administrativa, hacen que Desrosières considere la estadística un objeto de estudio especial. La estadística produce todo un mundo de cifras e indicadores que dan soporte a la realidad social, hablan de cosas que existen, pero que al mismo tiempo son convenciones surgidas de prácticas administrativas o políticas concretas. Este interesante análisis histórico nos invita a dirigir la mirada hacia el proceso de construcción de esas objetivaciones de la realidad social, deteniéndose en los procesos de definición, codificación y registro que acompañan a la medición, sometiendo a un análisis crítico a la razón estadística, considerada comúnmente como una autoridad. Los objetos estadísticos, aparentemente neutros, están presentes en las actividades científica, política y económica, y provienen, en primer lugar, de la construcción de un espacio político de equivalencia y codificación y, después, en un tratamiento matemático sustentado en el cálculo de probabilidades. Los vínculos inseparables entre la ciencia y la acción, requieren de un análisis conjunto de dos perspectivas históricas, la historia de las instituciones administrativas y políticas y la historia del conocimiento científico y los métodos matemáticos. Sólo desde esa doble perspectiva se puede dar cuenta del movimiento circular entre la ciencia y la acción.

El análisis sociológico de la estadística que hace Desrosières se extiende no sólo al contenido científico de las herramientas estadísticas sino también a los actores que lo hacen posible y las redes sociales donde están situados. Aunque la metodología empleada por Desrosières puede ser rastreada desde las enseñanzas de Bourdieu, en el sentido de suscitar el interés por las condiciones de producción del conocimiento científico, va más allá de las consideraciones sobre las « lentes de observa- ción» de los estadísticos y desciende al contenido mismo del conocimiento estadístico. Esta perspectiva metodológica está más cerca del programa fuerte de la sociología de la ciencia y más en concreto de los autores franceses $M$. Callon y $B$. Latour, de los que toma la idea del análisis de las controversias originadas en el contexto de la producción científica, desde la convicción de que estas controversias constituyen situaciones privilegiadas para observar el trabajo de los científicos. Sin embargo, Desrosières se distancia de las prácticas etno-metodologicas que se centrarían en la observación de los actores de la actividad estadística concreta; por el contrario busca en el análisis de la estadística una mirada más amplia que pueda, además, dar cuenta de las generalidades y objetivaciones, que trascienden el ámbito de los actores concretos. No sólo se interesa por la sociología de la estadística, sino también de lo que ésta nos enseña acerca de la sociedad.

Esta perspectiva, sitúa el contenido de la ciencia al mismo nivel que las condiciones sociales en las que se produce; a diferencia de las ciencias duras, la estadística como manifestación del conocimiento científico se presta especialmente a esta manera de verlas cosas. Como apunta Desrosières, si de alguna forma pudiera concebirse que los enunciados de la física fuesen independientes de las consideraciones sociales acerca de las personas o los laboratorios en los que se estudia, cuando se observa la sociedad mediante los instrumentos estadísticos no es posible establecer la separación entre las formalizaciones matemáticas y las redes sociales en las que se establecen.

Pero introducir la razón estadística dentro de una investigación histórica política y social, exige un cambio de mentalidad casi tan dificil para el investigador como para el ciudadano en general: es necesario vencer las resistencias debidas al hábi- 
to profundamente arraigado de comprender el mundo social a través de una densa red de índices y porcentajes. Sólo atendiendo a los cambios producidos en el razonamiento estadístico a través del tiempo, siguiendo las transformaciones de la estadística al hilo de la evolución de los Estados y las administraciones, podemos acceder a su verdadera esencia.

La estadística comienza como instrumento administrativo -se trataba de reunir toda la información que fuera relevante para el Estado- pero en cada país se produjo una estadística diferente. El análisis de Desrosières se extiende a Alemania, Francia Inglaterra y Estados Unidos. La inserción de las construcciones estadísticas en sus respectivos contextos le permite establecer que no hay una sola realidad objetiva, sino que las descripciones de la realidad social, orientadas a acciones políticas históricamente situadas, producen soluciones diferentes, desde la « statistik» alemana no cuantitativa, escrita en tablas de doble entrada, hasta la « aritmética política» inglesa, basada en cálculos matemáticos abstractos.

Pero la incorporación de los instrumentos matemáticos no es inmediata sino producto de un largo proceso. Para comprender el papel de esos cálculos matemáticos el autor nos introduce en el origen del cálculo de probabilidades. Desde la mesa de juego en el siglo XVII a la astronomía en el siglo XIX, y de aquí a las ciencias sociales y la econometría, el concepto de probabilidad es objeto de profundas controversias. Si bien el debate entre la probabilidad concebida como frecuencia relativa o como creencia subjetiva, no se resuelve hasta la síntesis de ambas tendencias en los trabajos de Gauss y Laplace. Con todo, la introducción del cálculo de probabilidades en la estadística del siglo XIX, supone un hito decisivo en la historia de la estadística. La aplicación de los modelos matemáticos de las mediciones astronómicas al campo de lo social, de la mano de Quetelet, inaugura una nueva forma de hablar de la sociedad: los agregados sociales. Las regularidades observadas en los agregados venían a poner orden en el caos de las manifestaciones individuales, siempre contingentes. Pero la existencia de un hombre medio, un hombre teórico que posee las características medias de toda una población, es objeto de intensos debates entre posiciones realistas y nominalistas acerca de esos objetos estadísticos. Estas generalizaciones serán uno de los puntos estratégicos donde Desrosières trate de reconstruir los pasos de la construcción de esa razón estadística. El establecimiento de los agregados sociales y la consolidación de su realidad social, tendrá una gran trascendencia en todos los campos. El análisis histórico permite abrir el campo de esos instrumentos descriptivos orientados a la acción, que por su propia naturaleza aparecen como algo especialmente sólido e incuestionable.

El proceso de construcción de objetos los estadísticos, tan familiares en la actualidad como los sondeos, nos ayuda a comprender su alcance $y$ trascendencia, abriendo de nuevo todo el proceso de discusión que modeló las formalizaciones. La necesidad de reunir datos sobre la totalidad a partir de sólo una parte, enfrentada al ideal del recuento exhaustivo, abre paso a diferentes significados de la idea de representatividad. Los estudios parciales de monografías centradas en casos típicos van dejando paso a los sondeos basados en una muestra representativa, primero elegida según el juicio del investigador, luego de forma sistemática y finalmente aleatoria. La posibilidad de conocer algo acerca de la totalidad a partir de una muestra representativa condensa toda una serie de factores sociales, administrativos, matemáticos, económicos e ideológicos.

Otro punto de especial interés en el trabajo de Desrosières es el de la codifica- 
ción y la nomenclatura, campo en el que aplica la experiencia profesional en el INSEE durante muchos años, especialmente en el campo de las categorías profesionales. Partiendo de los primeros sistemas de clasificación en el trabajo taxonómico aplicado a la naturaleza, hasta llegar a los sistemas de clasificación actuales. Este análisis es novedoso, pues si en la década de 1980 las herramientas matemáticas y las administraciones estadísticas fueron objeto de diversas investigaciones históricas, no ocurrió lo mismo con los procedimientos de clasificación y codificación. Este es un campo que ha permanecido oculto, pero por poco visible que resulte no deja de ser esencial y previo al establecimiento de las objetivaciones estadísticas. En el ámbito de las ciencias sociales, la taxonomía y la codificación son considerados problemas prácticos más que teóricos y a menudo se han resuelto en el ámbito de la práctica cotidiana, haciendo más hincapié en las condiciones administrativas y sociales que en una reflexión metodológica.

Si al principio la estadística fue tan sólo un producto de la actividad administrativa, en el XIX surge la estadística moral, fruto de las preocupaciones sociales en una época de grandes transformaciones. Ambas tendencias, la administrativa y la sociológica se condensan en la obra de Quetelet y su búsqueda de las leyes que rigen la sociedad. La perspectiva que unifica ambas perspectivas estadísticas será el origen, posteriormente, tanto de estadísticas que utilizarán las categorías de los registros administrativos construidas en el cotidiano trabajo con los datos, como de estadísticas basadas en categorías subyacentes puestas en evidencia por las regularidades observadas en el tratamiento matemático de los datos. Todo esta problemática es tratada a partir de cuestiones concretas como la aparición de nuevas categorías sociales. La evolución de las definiciones estadísticas, como el paso desde la categoría de « pobre» a la de « parado", debida a un cambio en la óptica de las políticas sociales -en este caso, la evolución desde una política social basada en la caridad, a otra centrada sobre todo en la regulación del trabajo- nos recuerda el sentido olvidado de las categorías con las que hablamos de la sociedad. El análisis de la categorización en clases sociales en los estudios sobre la pobreza en Inglaterra ofrece todo un mundo de debates centrados en la manera de definir el problema. Si la administración que presta los servicios sociales utiliza categorías orientadas a la acción política y relacionadas con el medio, como el lugar de residencia o la situación laboral, las corrientes teoricas partidarias de la eugenesia se muestran críticas con lo que consideran " caridad contraproducente» y se afanan en el proyecto de definir el problema en términos de herencia genética, construyendo sus categorías en términos de características hereditarias individuales. Asimismo, los debates en torno a la clasificación y codificación de las enfermedades o las causas de la muerte, desde los puntos de vista etiológico, centrado en las causas, o topológico, orientados hacia los síntomas, dan cuenta del proceso en el que las formalizaciones estadísticas adquieren valor como argumentos en el debate y de la forma en que se construye el consenso a la hora de definir una situación concreta. La cuestión de la categorización y codificación de los datos queda siempre abierta al debate $y$ es especialmente sensible a las determinaciones, tanto políticas y teóricas como económicas y prácticas. Desrosières muestra en este ámbito cómo es posible sostener un debate sobre esos objetos estadísticos « indiscutibles».

La utilización de las estadísticas en el terreno económico, ilustra especialmente la complejidad de corrientes teóricas, muchas veces contradictorias, que conver- 
gen en la economía matemática y luego en la econometría. El recurso simultáneo a la matematización, la cuantificación y el cálculo de probabilidades, no resulta evidente por sí mismo. El enfrentamiento entre el pensamiento hipotético-deductivo de la economía teórica y el descriptivo-inductivo de la economía histórica, se resuelve en una síntesis que abandona el campo de la filosofía política y las disciplinas literarias para entrar en el terreno de las matemáticas. Aunque la economía matemática del XIX pretendía reducir la complejidad del universo económico a un número limitado de leyes que permitieran deducir un modelo lógico y coherente, se seguían produciendo datos provenientes de la acción administrativa, los registros y los agregados estadísticos. El recelo de los estadísticos descriptivos hacia los modelos controlados por un número limitado de variables, es comparable al que manifiestan los economistas matemáticos hacia las estadísticas administrativas que reúnen datos sobre hechos complejos ajenos a sus modelos. Serán precisamente las herramientas matemáticas surgidas en otros campos, como la eugenesia o la teoría cinética de los gases, las que posibilitarán el deslizamiento desde la estadística administrativa o moral, a la estadística matemática. La correlación y la regresión, permitirán el acercamiento de ambas tendencias, pero no será hasta la introducción del cálculo de probabilidades en la economía cuando las formalizaciones matemáticas y los datos empíricos se reconcilien. Herramientas tan comunes en la actualidad como la inferencia estadística a partir de un número limitado de observaciones, surgen de esta difícil síntesis. La diferencia conceptual entre un parámetro objetivo de la población y su estimación estadística pertenecen a esta lógica. Las posiciones enfrentadas sobre la naturaleza de las probabilidades, una objetiva basada en las frecuencias observadas, y la otra subjetiva, definida como estado mental y grados de creencia o desconocimiento, se acercan en un modelo teórico que permite tanto la estimación subjetiva acerca de un suceso - ya sea regular o único- como la realización de test sobre hipótesis sustentadas en una en una concepción frecuentista.

El ejercicio propuesto por Desrosières, ese cambio de mentalidad necesario para discutir sobre las estadísticas que nos describen la realidad, nos permite de alguna forma desmitificarlas y tener una visión más profunda de esos objetos estadísticos que, precisamente por ser cotidianos, aparecen cerrados como cajas negras. El análisis histórico de la génesis y evolución de la razón estadística nos ayuda a comprender de manera crítica el alcance de las trasformaciones en la forma de ver la sociedad a través de las representaciones mediante conceptos, índices, tablas y gráficos. La política de los grandes números se convierte así en un texto esencial, tanto para los políticos, economistas o sociólogos, como para el ciudadano en general, inmersos en un mundo de cifras cuyo sentido ha sido a menudo olvidado.

Alejandro Almazán 